\title{
Amélioration des performances hors adaptation d'une pompe axiale
}

\author{
Improvement of pump performance \\ at off design conditions
}

J. Paulon

Office national d'études et de recherches aéronautiques

BP 72, 92322 Châtillon Cedex

C. Fradin

\author{
J. Poulain \\ Alsthom \\ 141 , rue Rateau \\ BP 111, \\ 93123 La Courneuve
}

Les pompes industrielles sont généralement utilisées dans un large domaine de fonctionnement, depuis un débit presque nul jusqu'à des débits sensiblement plus élevés que la valeur de projet.

Souvent, on a observé que la caractéristique hauteur-débit, à vitesse constante, présente un "creux" négatif. lorsque le débit devient légèrement inférieur au débit de projet. Des instabilités aérodynamiques et mécaniques apparaissent, qui peuvent être dangereuses pour la machine.

Afin d'analyser et, si possible, de pallier ces difficultés, une étude expérimentale a été entreprise conjointement par l'ONERA et Alsthom/Rateau. Une analyse détaillée de l'écoulement a mis en évidence des décollements et d'importants effets tridimensionnels.

De cette meilleure connaissance du champ d'écoulement, nous avons pu imaginer un dispositif particulier qui a permis d'obtenir une très forte atténuation du "creux ".

Industrial pumps are generally used in a wide range of operating conditions from zero or almost zero mass flow to mass flows larger than the design value. It has been often noted that the head-mass flow characteristic of conventional axial flow pumps operating at constant speed presents a negative hump as the mass flow becomes somewhat smaller than the design mass flow.

The pump does not stall, there is no real low frequency oscillations in the circuit, but flow and mechanical instabilities, unsafe for the facility, are present for some intermediary flow range.

A joint experimental venture between ONERA and Alsthom/Rateau Division was undertaken in order to analyze and if possible to palliate these difficulties.

The tests were performed in air instead of water. keeping the same flow coefficients.

\section{Introduction}

L'utilisation industrielle des pompes nécessite généralement de fonctionner dans un large domaine de débit, depuis un débit quasi nul jusqu'à une valeur maximale nettement plus grande que la valeur projet.

Expérimentalement, on observe souvent sur les pompes axiales que la caractéristique hauteur de refoulement-débit présente un "creux" pour des débits intermédiaires légèrement inférieurs au débit de projet $[1,2]$. Un exemple typique d'une telle caractéristique est donné sur la figure 1 ; lorsque le débit décroît, la hauteur croît pour atteindre une valeur maximale. Après une légère diminution de la hauteur avec le débit, la hauteur augmente à nouveau, tandis que le rendement décroît rapidement.

Un tel type de fonctionnement présente deux inconvénients : 


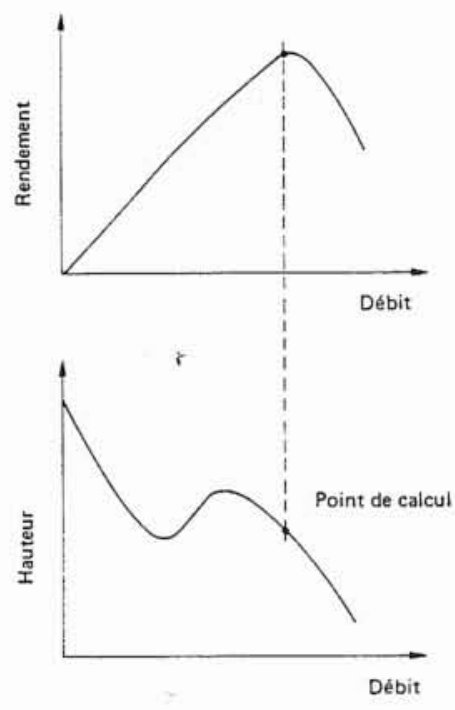

Figure 1. - Allure de la variation de la hauteur et du rendement d'une pompe axiale en fonction du débit.

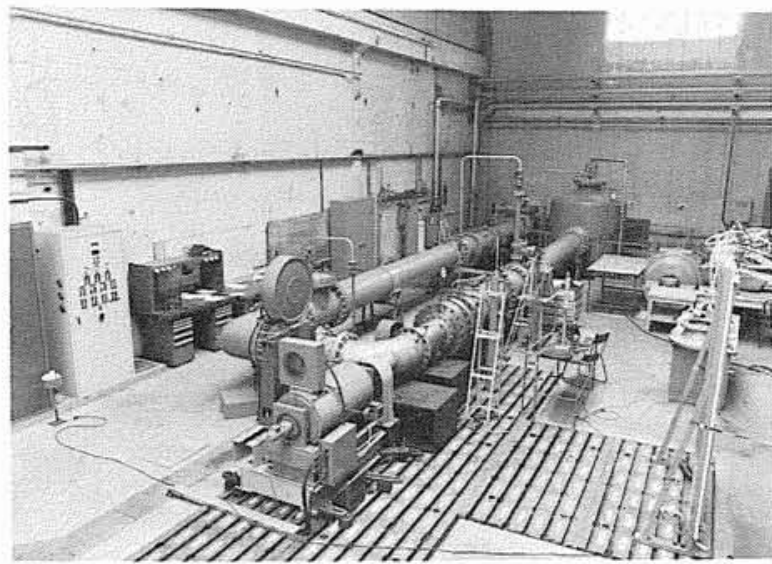

Banc d'essais en eau

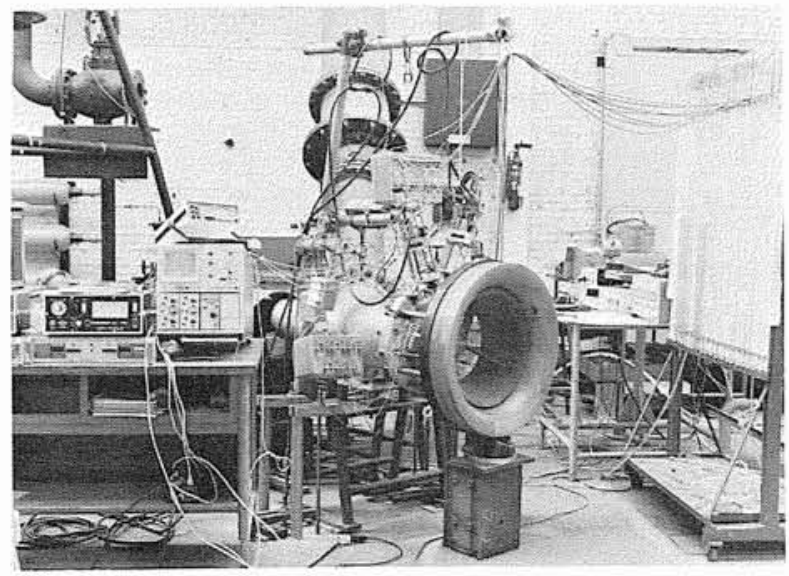

Banc d'essais en air

Figure 2. - Vue des bancs d'essais.
Notations :
$D=$ débit volume, $\mathrm{m}^{3} \cdot \mathrm{sec}^{-1}$
$g=$ accélération de la pesanteur, $9,81 \mathrm{~m} \cdot \mathrm{sec}^{-2}$
$H=$ hauteur de refoulement, $\mathrm{m}$
$R=$ rayon externe, $\mathrm{m}$
$U=$ vitesse périphérique, $\mathrm{m} \cdot \mathrm{sec}^{-1}$
$\delta=$ coefficient de débit
$\mu=$ coefficient manométrique de hauteur

- lorsque la hauteur de refoulement décroît avec le débit, des instabilités apparaissent, qui peuvent induire de fortes vibrations et qui parfois peuvent conduire à la détérioration de certains éléments;

- le domaine utile de fonctionnement se trouve réduit par rapport au domaine théorique.

Avec l'aide du ministère de la Recherche (M.R.T.), une étude expérimentale a été entreprise conjointement par I'ONERA et la Division Rateau d'Alsthom, dans le but d'éliminer ou au moins de réduire le «creux".

Les mesures ont été commencées en eau mais poursuivies en air, pour des raisons de facilités expérimentales. On a cependant vérifié que les coefficients de débit et de hauteur manométrique étaient les mêmes dans les deux cas.

A partir d'une analyse détaillée du champ d'écoulement dans différentes sections de la pompe et pour différents débits, une meilleure connaissance de ce champ a pu être obtenue et une configuration particulière, qui permet de réduire notablement le "creux ", a été expérimentée.

\section{Montage expérimental}

Les figures $2 a$ et $2 b$ montrent une vue des bancs d'essais en eau et en air. La pompe axiale se compose d'une roue de quatre aubes, dont le calage peut être modifié à l'arrêt, et d'un redresseur de sept aubes. Les résultats présentés se rapportent au seul calage du projet.

Le diamètre extérieur est de $296 \mathrm{~mm}$ et le diamètre intérieur de $120 \mathrm{~mm}$. Les carters interne et externe sont cylindriques. Le nez central, en amont de la roue, est fixé par trois bras au carter externe, ce qui permet le passage de lignes de mesure de pression.

Une vue méridienne de la pompe et l'emplacement de l'instrumentation de mesures sont montrés sur la figure 3; on note :

- 10 prises classiques de pression statique sur le carter externe (repérées de 1 à 10), le long d'une génératrice;

-8 prises du même type (repérées $\mathrm{A}$ à $\mathrm{H}$ ), également sur le carter externe, mais disposées sur une ligne moyenne des aubes, en tête de la roue;

- 6 prises classiques (repérées 11 à 31 et 81 à 101) disposées sur une génératrice du moyeu, de part et d'autre de la roue mobile;

- 4 orifices pour le passage de sondes de pression ou de fils chauds (repérés I à IV).

Enfin, un carter externe transparent permet une observation directe de l'écoulement au niveau de la roue mobile. 


\section{Résultats des mesures et interprétation}

\subsection{Caractéristique hauteur de refoulement-débit}

Sur la caractéristique sans dimension de la figure 4, où le coefficient de hauteur $\mu=g h / U^{2}$ est représenté en fonction du coefficient de débit $\delta=D / U R^{2}$, le "creux " est nettement visible.

Sur cette figure, on a également indiqué les valeurs du débit (repérées de 1 à 7) pour lesquelles des analyses détaillées ont été effectuées.

\subsection{Répartitions de pression statique sur le carter externe}

Les répartitions de pression statique relevées sur le carter externe pour les différents essais sont représentées sur la figure 5 .

Les essais 1 et 2 correspondent à des fonctionnements en régime sain, et on note l'accélération de l'écoulement en amont de la roue, caractérisée par la pente négative du coefficient de pression statique.

Pour les essais 3 et 4 , qui correspondent à la partie négative du " creux " l'accélération précédente s'atténue, ce qui tend à montrer la présence d'une recirculation près du carter externe. La partie aval des répartitions de pression correspondant à ces quatre essais est pratiquement la même, puisque le coefficient de hauteur varie peu.

Cette recirculation périphérique s'étend de plus en plus en amont, au fur et à mesure que le débit diminue (essais 5 à 7 ) et la pression aval croît.

Le fait qu'il y ait un maximum de pression dans la roue peut expliquer le fait, détaillé plus loin, que des effets centrifuges apparaissent dans la roue au voisinage des sections de tête.

\subsection{Explorations d'écoulement par sonde directionnelle}

A l'aide de sondes directionnelles, des répartitions radiales de la pression totale, de la pression statique et de la direction de l'écoulement (avec l'axe de la machine) ont été obtenues; il n'y a pas eu de mesure de pente de l'écoulement.

Ces mesures, effectuées en plusieurs plans et validées par de bons recoupements en débit, ont permis de schématiser les configurations d'écoulement, comme le montre la figure 6. Comme prévu, pour les essais 1 et 2 , l'écoulement méridien est pratiquement uniforme. Pour l'essai 3, à débit plus faible, on observe l'apparition d'une petite zone de recirculation en tête et en amont de la roue mobile. En même temps, un décollement prend naissance au pied, à la sortie de la roue.

Ces deux phénomènes prennent de l'ampleur, au fur et à mesure que le débit diminue (essais 4 et 5 ) et un décollement apparaît en tête dans le redresseur pour un débit encore plus faible (essai 6).

A débit quasi nul (essai 7), on constate que l'écoulement est pratiquement radial à l'intérieur de la roue, tandis que l'on note deux tourbillons contrarotatifs à la sortie du redresseur.

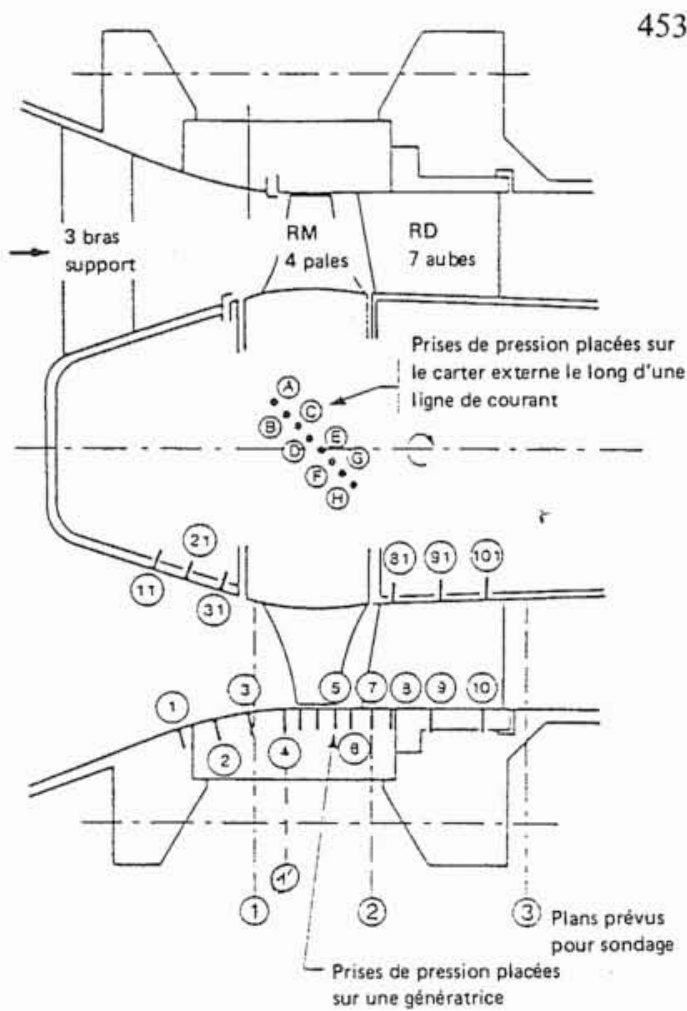

Figure 3. - Vue méridienne de la pompe et instrumentation.

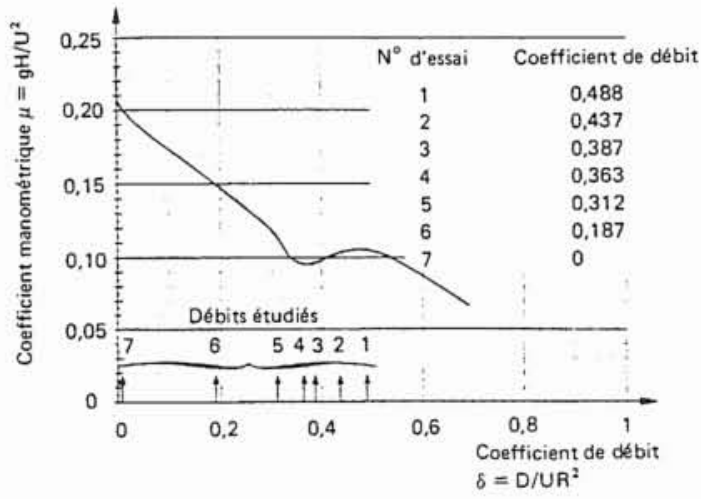

Figure 4. - Caractéristique non dimensionnelle hauteur-débit de la pompe.

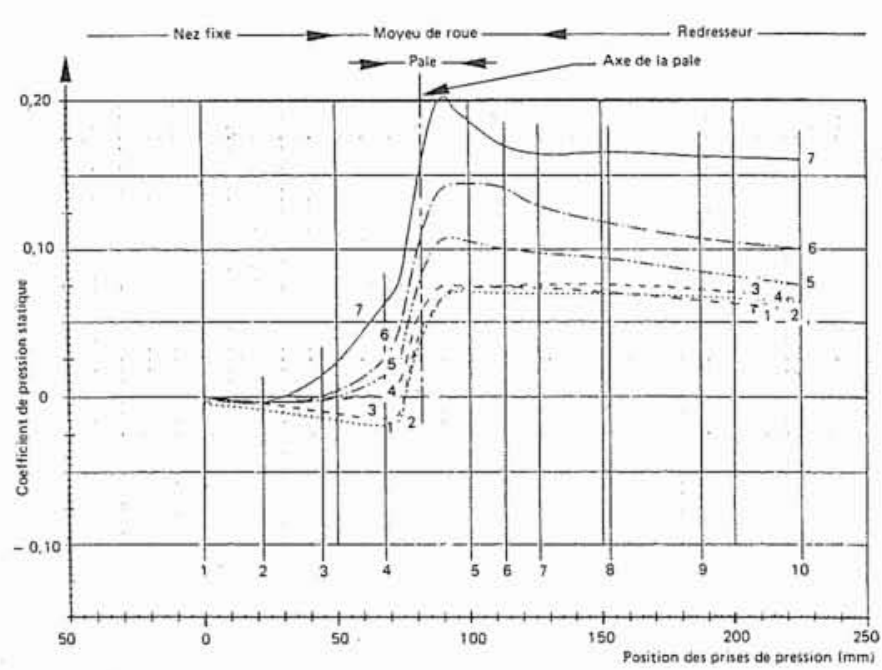

Figure 5. - Répartition des pressions pariétales. 



Figure 6. - Configurations d'écoulement déduites des sondages radiaux : schemas d'écoulement dans la pompe

a) Régime sain, b) Point de fonctionnement au début du "creux " de la caractéristique. c) Poin de fonctionnement dans le "creux" de la caractéristique. d) Point de fonctionnement à la sortie du «creux" de la caractéristique. e) Point de fonctionnement a faible débit. f) Poinl de fonctionnement proche du débit nul.
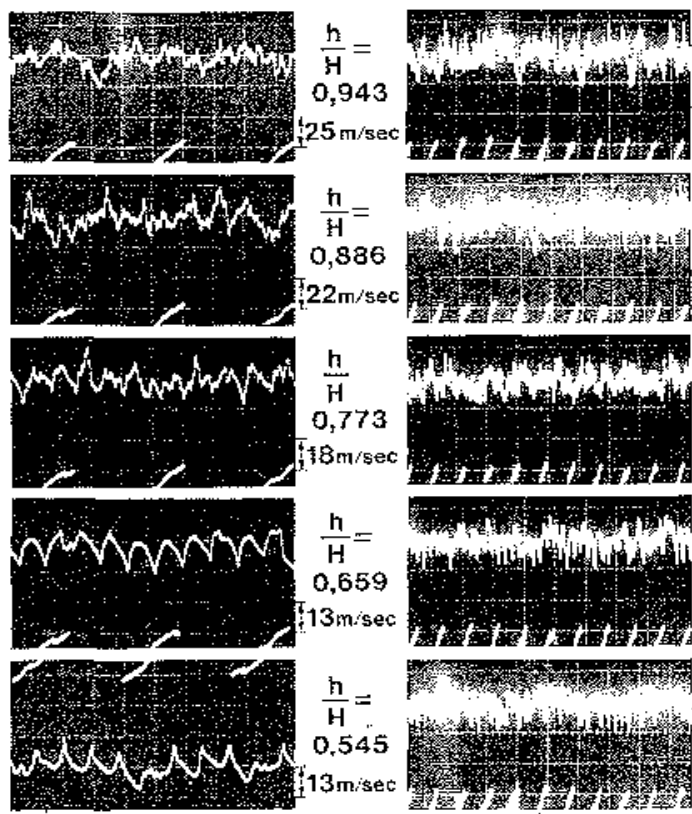

1 tour de roue

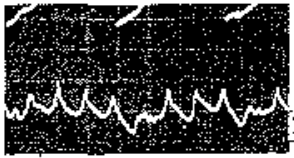

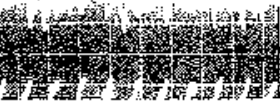
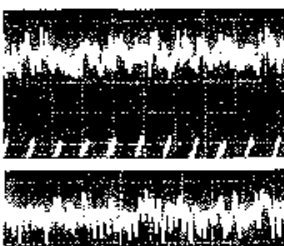

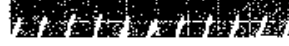
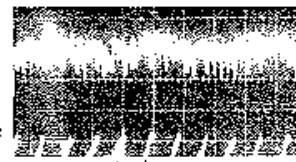

1 tour de rove

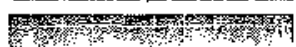

Figure 7. - Mesures par fil chaud en amont de la roue mobile (essai 4).

\subsection{Explorations d'écoulement par fil chand}

Les fluctuations de pression au niveau des parois étant trop faibles pour être correctement mesurées par des capteurs, des mesures par fil chaud ont été effectuées de part et d'autre de la roue. La figure 7 montre un exemple de résultats obtenus en amont de la roue pour l'essai 4 (situe dans le " creux »).

Sur la partie gauche de la figure, la perturbation due au passage des quatres aubes est très nette pour des valeurs de la hauteur relative $h / H$ inférieures à environ 0,75. Au-dela, un écoulement perturbé et turbulent apparaît ( $h=0$ au moyeu).

Sur la partie droite de la même figure, représentant les mêmes essais qu'à gauche, mais avec une autre base de temps, on n'observe pas de fluctuations périodiques du type décollement tournant. Ceci est assez normal, compte tenu du faible nombre d'aubes de la roue.

De même, il n'a pas été observé de pompage à très faible débit, ce qui peut être expliqué par le faible rapport de pression de la machine.

\section{Réduction du "creux"}

Actuellement, il n'existe pas de modèle théorique pour décrire des champs d'écoulement aussi compliqués que la plupart de ceux représentés sur la figure 6. L'amélioration des pompes doit être obtenue, dans ces cas, par des moyens expérimentaux. Quelquesuns ont été suggérés dans la littérature [3].

Puisquapparemment les difficultés sont liées aux faibles débits et à des recirculations, on peut espérer trouver des remèdes en jouant sur l'un ou l'autre phénomène.

L'emploi d'un diaphragme pour accroitre la vitesse méridienne à l'entrée d'une roue est une des solutions envisageables. Un exemple de diaphragme réduisant de $20 \%$ la section de passage est indiqué sur la figure 8a. Les performances correspondantes présentées sur la figure $8 b$ que le «creux" a disparu, mais avec l'inconvénient de pertes de performances. Ce n'est donc pas une bonne solution.

Un baffle, constituant une séparation cylindrique entre le moyeu et la tete, peur modifier la recirculation, au moins en amont de la roue. La figure $9 a$ montre les positions des deux baffles essayés : le baffle $B 1$ divise la section d'entrée en deux parties ègales, tandis que le baffle B2 est placé approximativement au centre de la zone de recirculation. La figure $9 b$ montre que le baffle B2 est le plus efficace, puisqu'il augmente même les performances de la pompe aux faibles débits sans les détériorer pour les débits élevés.

Pour tenter d'expliquer l'efficacité de tels baffles, des explorations radiales ont été effectuées. La figure $10 a$ montre que sans le baffle, le coefficient de pression reste constant le long du rayon à l'entrée de la roue, aussi longtemps que les valeurs du coefficient de débit sont élevées (essais 1 et 2 de la figure 4), mais il croît 

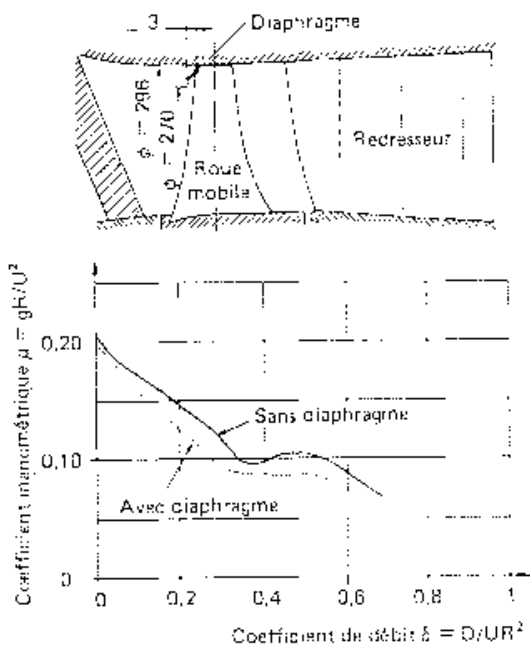

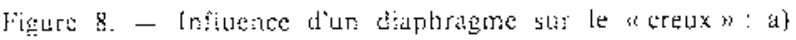

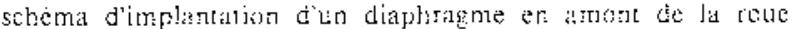
mobile. b) caractérisi ique $\mu-\delta$ ce ja porme
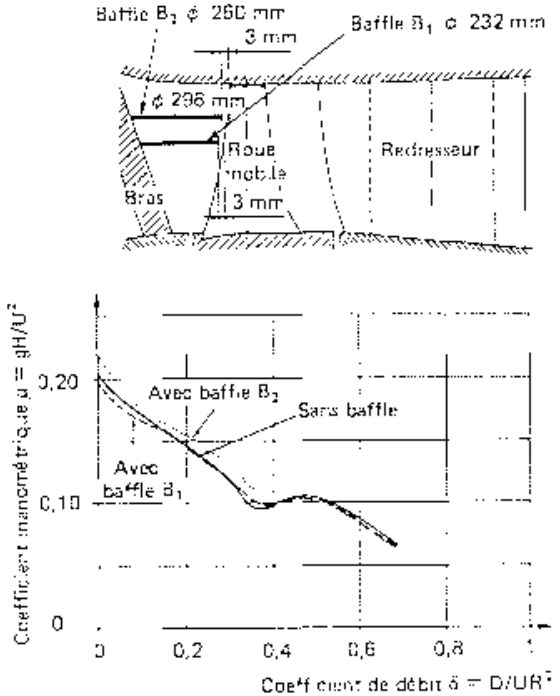

Fïgute 9. Infïuence dun baffle suc le «creux» : aj schema d'implantation ce baffles en amont de la roile mobile, j) catractcrist:ụue $\mu-\delta$ de lá portipo
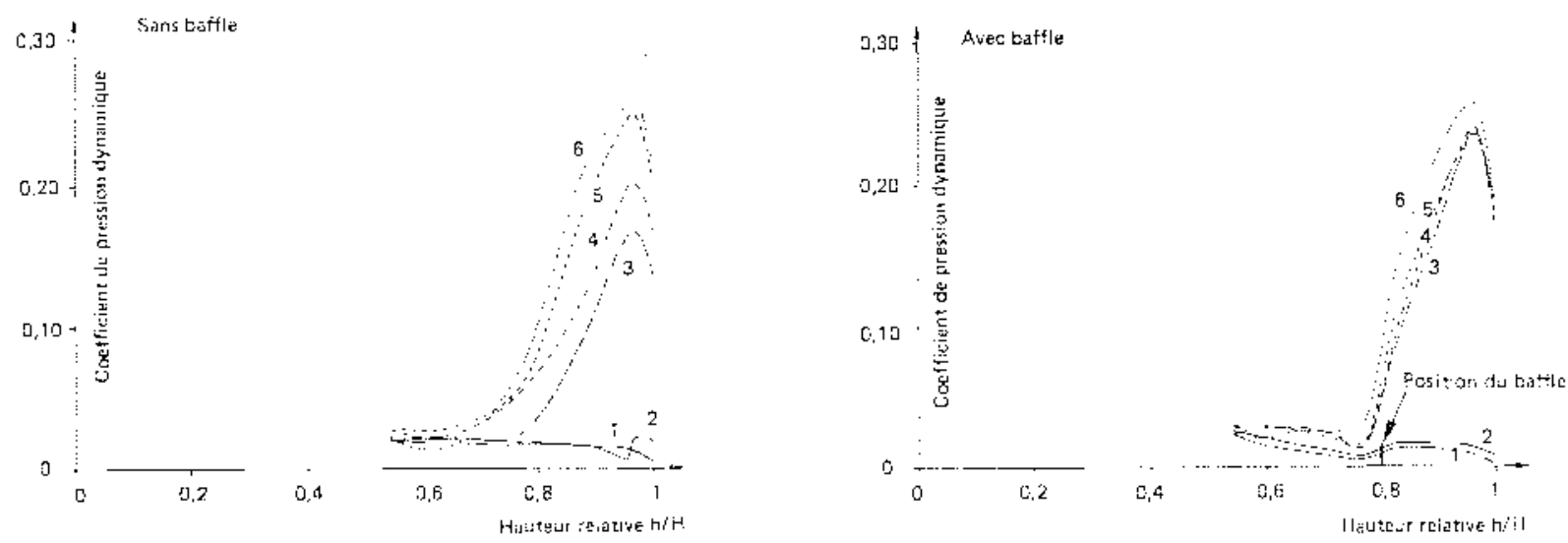

Figure 10. - Distribution rixíale du ceetrictent de prossion

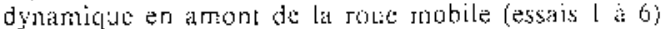

progressivement près du carter externe lorsque le débit diminue (esisitis 3 à 6).

Rien ne change pour les essais $1 \mathrm{ct} 2$, lorsque le batfle est mis en place, mais aussitot qu tine recirculation apparaît en tête (cssais 3 à 6), lat répartition du coefficient de pression prend une allure quasi unique, indépendante du débit ffgure 10b). Apparement, l'amélioration de performance doit ctre attribué à la meilleure uniformité de l'écoulement central.

\section{Visualisations}

Des visualisations ont été fátes à l'àde de fìls de laine colles sur les bras support du nez.

Comme le montre la figure $/ l$, le baffle ne modifie pas le champ d'écoulement à grand débit (essai 1), tandis qu'à plus fafble (fébit (esミai 4) la recirculation, qui n'apparaissait pas en l'absence du bafle, devient très neate cn présence de ce dernicr. 


\section{Conclusion}

Le " creux " de la caractéristique hauteur-débit de certaines pompes axiales, qui limite le domaine d'utilisation de ces pompes, peut être réduit ou même supprimé en employant un baffle en amont de la roue mobile. Un tel dispositif n'a pas d'effet négatif notable sur les performances à grand débit et améliore même celles à petit débit.

Le " creux » est dû à l'apparition de recirculation et on constate qu'un baffle placé sensiblement au centre de cette recirculation a des effets de contrôle et de régulation bénéfiques.

\section{Références}

[1] TANAKA T. - An experimental study of diagonal flow propeller pump : design performance with various types of blading, A.S.M.E. paper 77 W.A./F.E. 9, Atlanta, Nov. 24th-Dec. 2nd, 1977.

[2] TANAKA T. - An experimental study of backflow phenomena in a high specific speed propeller pump, A.S.M.E. paper 80 F.E. 6, New Orleans, March 10-13th, 1980.

[3] MURAKami M., HeYa N. - Improvement of pump performance by impeller eye throttling. A.S.M.E. paper 69 F.E. 26, Evanston, June 16-18th, 1969.
Essai 1

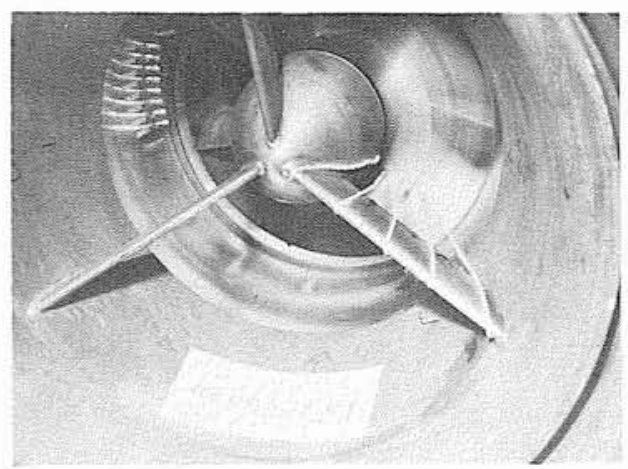

Essai 4

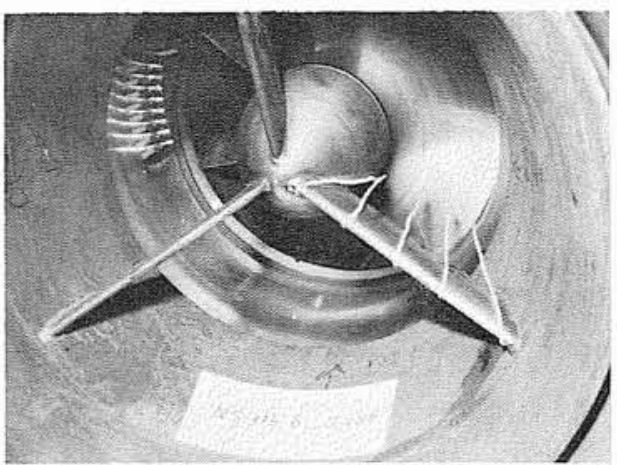

sans baffle
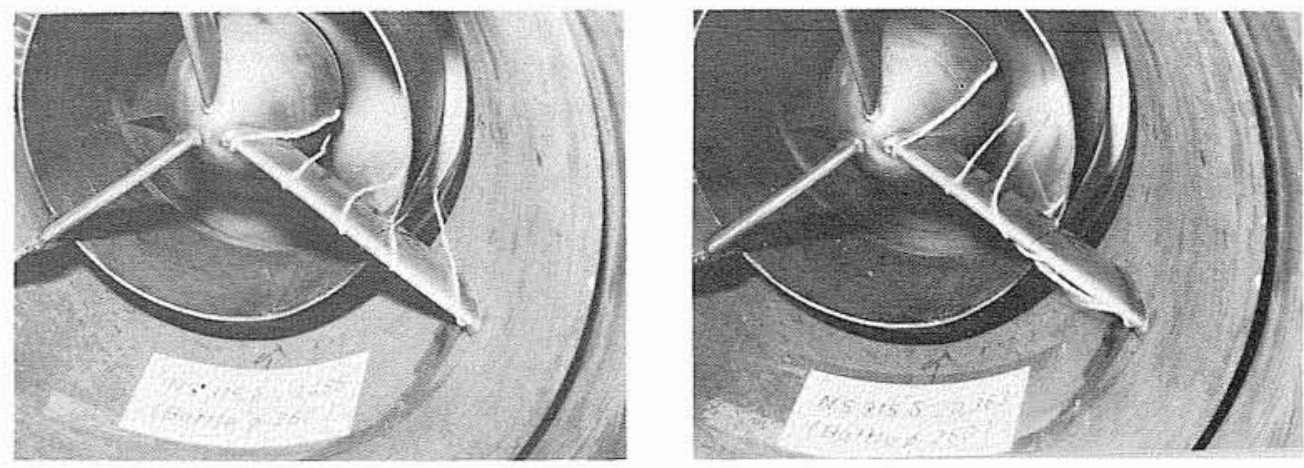

\section{avec baffle}

Figure 11. - Visualisation par fils de laine de l'écoulement amont. 